\title{
Fatal Central Nervous System Lymphocytic Vasculitis after Treatment for Burkitt Lymphoma in a Patient with a SH2D1A Mutation
}

\author{
João Farela Neves, MD, *† Lamberto Torralba Raga, PhD, $\neq$ Samuel C. C. Chiang, PhD, $\neq$ Bianca Tesi, PhD, $\$$ \\ José Pedro Vieira, MD, Il Ana Isabel Cordeiro, MD, * Luis Borrego, PhD, ${ }^{* * *}$ and Yenan T. Bryceson, PhD $\neq$
}

\begin{abstract}
Very rarely, patients with X-linked lymphoproliferative syndrome type 1 present central nervous system vasculitis. We report a patient carrying a SH2D1A mutation that, after treatment for lymphoma developed fatal central nervous system vasculitis. He lacked signs of ongoing EpsteinBarr virus infection. We propose that impaired $\mathrm{T}$ cell homeostasis caused by $\mathrm{SAP}$ deficiency facilitates aberrant $\mathrm{CD} 8^{+} \mathrm{T}$ cell activation against vascular antigens promoting clinical manifestations.
\end{abstract}

Key Words: XLP1, SAP, CNS vasculitis, lymphoma, EBV, SH2D1A

(Pediatr Infect Dis J 2019;38:e29-e31)

$X$ -linked lymphoproliferative syndrome type 1 (XLP1) is a rare primary immunodeficiency with an estimated incidence of 2 males/1.000.000 newborns. ${ }^{1}$ XLP1 is caused by hemizygous mutations in SH2D1A, located at Xq25, that encodes the signaling lymphocytic activation molecule (SLAM)-associated protein (SAP). ${ }^{2}$ $\mathrm{SAP}$ is a cytoplasmic protein expressed in NK and T cells. Upon recruitment to SLAM family receptors (e.g., 2B4), SAP promotes activating signaling. SAP deficiency renders SLAM receptors on lymphocytes inhibitory, resulting in impaired $\mathrm{NK}$ and $\mathrm{CD} 8^{+} \mathrm{T}$ cell cytotoxic responses toward SLAM family receptor-expressing hematopoietic target cells. ${ }^{2,3}$ Notably, $\mathrm{CD}^{+} \mathrm{T}$ cell cytotoxic responses in XLP1 are mostly preserved when antigens are presented by tissue cells as well as dendritic cells, but compromised in response to many hematopoietic cells, including B cells. Accordingly, patients are particularly susceptible to Epstein-Barr virus (EPV), which establishes latency in B cells. ${ }^{4}$ During EBV infection, infected $\mathrm{B}$ cells upregulate expression of CD48 that binds $2 \mathrm{~B} 4$ expressed on T and NK cells. In XLP1 patients, impaired killing of EBV-infected cells may culminate in fulminant mononucleosis

Accepted for publication May 21, 2018.

From the *Department of Pediatrics, Primary Immunodeficiencies Unit, Hospital Dona Estefânia- CHLC, EPE; †Department of Pediatrics, CEDOC, Chronic Diseases Research Center, NOVA Medical School, Lisbon, Portugal; tCentre for Hematology and Regenerative Medicine, Department of Medicine, Karolinska Institute, Karolinska University Hospital Huddinge; §The Childhood Cancer Research Unit, Department of Women's and Children's Health, Karolinska Institute, Karolinska University Hospital Solna; ๆThe Clinical Genetics Unit, Department of Molecular Medicine and Surgery, and Center for Molecular Medicine, Karolinska Institute, Karolinska University Hospital, Stockholm, Sweden; \|Department of Pediatrics, Neurology Service, Hospital Dona Estefânia- CHLC, EPE, Lisbon, Portugal; and **Hospital CUF Descobertas, Immunoallergy Department, Lisbon, Portugal.

A written informed consent from the patient's mother has been obtained.

The authors have no funding or conflicts of interest to disclose.

Address for correspondence: João Farela Neves, ORCID 0003-3963-2331, Infectious Diseases Unit, Primary Immunodeficiencies Unit, Hospital Dona Estefania, Pediatric University Hospital, Rua Jacinta Marto, 1169-045 Lisbon, Portugal. E-mail: joao.farelaneves@chlc.min-saude.pt.

Supplemental digital content is available for this article. Direct URL citations appear in the printed text and are provided in the HTML and PDF versions of this article on the journal's website (www.pidj.com).

Copyright (C) 2018 Wolters Kluwer Health, Inc. All rights reserved.

ISSN: 0891-3668/19/3802-0e29

DOI: $10.1097 /$ INF.0000000000002154 with excessive release of cytokines. Without treatment, a majority of the patients ultimately die..$^{2,5}$

Another frequent presentation of XLP1 is hypo or dysgammaglobulinemia, secondary to defective interactions between $\mathrm{B}$ cells and follicular helper T cells. ${ }^{1,3}$ One fifth of XLP1 patients present with lymphoma, while a minority of them present other manifestations such as aplastic anemia or vasculitis. ${ }^{1}$

We report an XLP1 patient who developed fatal central nervous system (CNS) vasculitis in the absence of active EBV-infection, 6 months after completing treatment for Burkitt lymphoma.

\section{MATERIALS AND METHODS}

\section{Flow Cytometry}

For the evaluation of lymphocyte subsets, peripheral blood was analyzed by Flow Cytometry in a 4-color BD FACS Calibur (BD, San Jose, Calif.), using the BD IMK kit with Trucount tubes (BD Biosciences) according to the manufacturer's instructions. An additional panel of monoclonal antibodies (including CD45RA, CD45RO, CD62L, and HLA-DR) was also performed for further characterization of T-cell differentiation, using a lyse-wash protocol. Finally, the IOTest Beta Mark kit (Immunotech SAS, a Beckman Coulter Company, Marseille, France) was also used for the characterization of the TCR V $\beta$ repertoire.

Flow cytometry data analysis was performed using Multiset and CellQuestPro software (BD Biosciences).

\section{Antibody Response Evaluation}

Immunoglobulins were quantified in serum. Immunoturbidimetria was used to quantify $\operatorname{IgG}, \operatorname{Ig}$ A and $\operatorname{IgM}$. As for specific responses, antibodies for Diphtheria, Tetanus, and Pertussis were evaluated by enzyme-linked immunosorbent assay.

\section{SAP Expression}

Rested peripheral blood mononuclear cells (PBMCs) from the patient and controls were surface labeled for CD3 (OKT3), CD8 (SK1), CD19 (HIB19) and CD56 (NCAM16.2) before fixed in $4 \%$ paraformaldehyde and intracellularly stained for SAP (1C9, Abnova). Cells were acquired on an LSRFortssa (BD Bioscience) and analyzed with Flowjo 9.9.

\section{Western Blot}

Freshly isolated PBMCs were lysed in RIPA buffer (Santa Cruz Biotechnology) before protein content was quantified using a standard Bradford assay (ThermoFisher). Equal concentrations were run on a $12 \%$ Bis-Tris gel (Invitrogen) in the presence of $10 \mathrm{mM}$ DTT. Anti-SAP (1C9) from Abnova and rat anti-SAP (1D12) from Cell Signaling Technology were used for immunoblotting. Mouse antiactin (C4) from MP Biomedicals was used as the loading control.

\section{Polymerase Chain Reaction}

One million PBMCs from a healthy control and the patient were harvested for generating cDNA (M-MLV, Invitrogen) following 
manufacturer's protocol. Taq polymerase (Invitrogen) was used for the polymerase chain reaction (PCR) reaction. Primers synthesized: $\beta 2$-microglobulin forward 5' AGTATGCCTGCCGTGTGAACC 3'; $\beta 2$-microglobulin reverse 5' TGCGGCATCTTCAAACCTCCA 3'; SH2D1A exon 1/2 5' CCTATGTGTGCTGTATCACGG 3'; SH2D1A exon 3 5' TGGCTTCTGAAATGCTGAAATG 3'.

\section{RESULTS}

A previously healthy 14-year-old boy presented with abdominal pain and loss of weight and was diagnosed with sporadic ileocaecal Burkitt lymphoma (Fig. 1A and fig., Supplemental Digital Content 1, http://links.lww.com/INF/D232). No evidence of ongoing EBV infection was found, as PCR for EBV in the blood was negative and serology provided evidence of past exposure (VCAIgM negative, VCA-IgG positive, negative EBNA). His brother had died of fulminant mononucleosis at 2 years of age, prompting further investigation. The patient manifested hypogammaglobulinemia, reduced class-switched memory B-cells, and lack of NKT cells (Table, Supplemental Digital Content 2, http://links.lww. com/INF/D233). Moreover, SAP expression was absent, as measured by Western blot and flow cytometry in PBMCs (Fig. 1B, C).

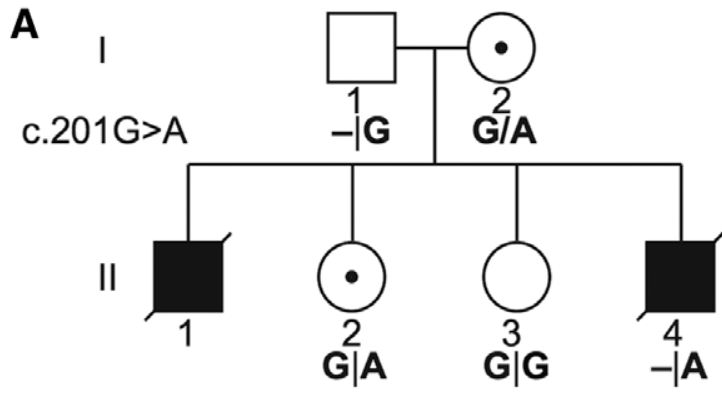

B

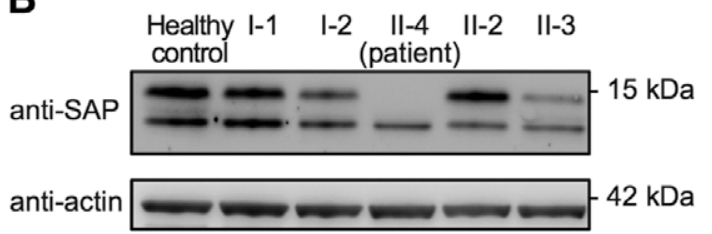

C

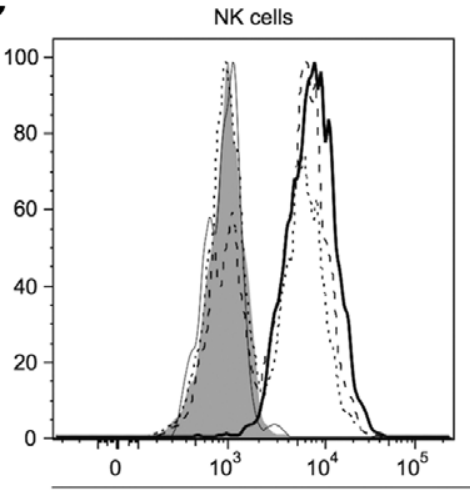

CD8+

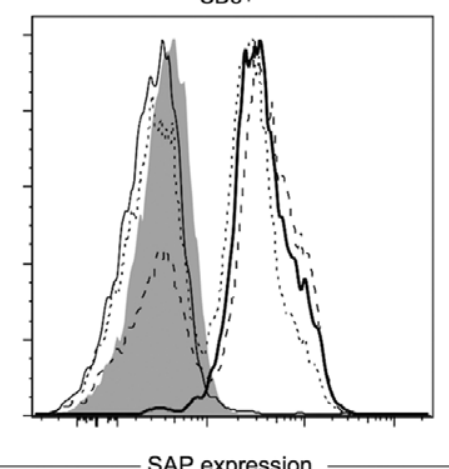

CD8+CD57+

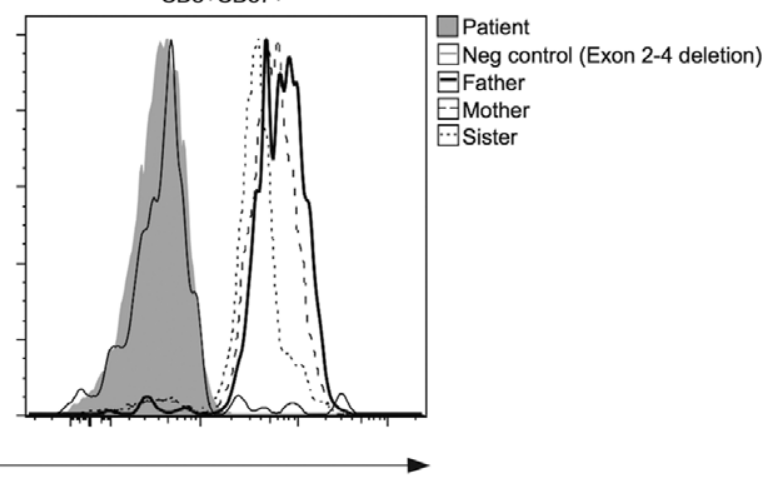

D

Healthy control II-4 (patient)

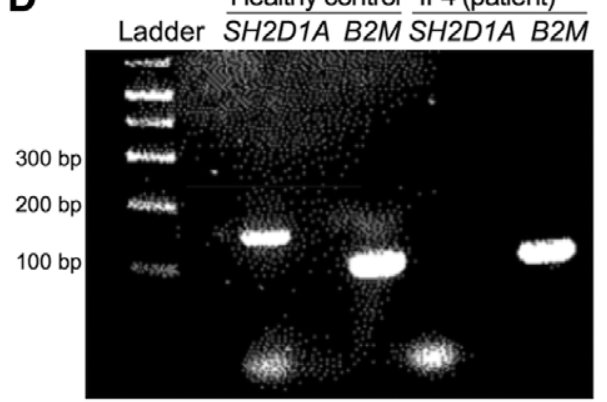

FIGURE 1. Pedigree and molecular diagnostics. A: Pedigree of the family including SH2D1A genotype. B: Analysis of SAP expression in PBMC lysates by Western blot in family members. C: Flow cytometric evaluation of SAP expression in NK and T cell subsets in controls and family members, as indicated. D: Sanger sequencing traces covering the patient SH2D1A mutation. E: PCR on PBMC-derived CDNA from a healthy control as well as the index patient using primers for SH2D1A and B2M, as indicated. 
Congruently, a hemizygous $S H 2 D 1 A$ c.201G $>$ A (p.E67E) synonymous mutation in exon 2 was identified (Fig. 1D), disrupting splicing of exon 2 as evaluated by reverse transcription-PCR (Fig. 1E). The lack of exon 2 in $S H 2 D 1 A$ mRNA results in a SAP protein lacking the crucial SH2 domain, as previously described by Debeljak et al. ${ }^{6}$

The patient was treated according to the mature B cell lymphoma 96 (LMB 96) protocol and achieved complete remission. The patient was proposed for hematopoietic stem cell transplantation (HSCT), but 6 months after completing the lymphoma treatment, he presented with a short-term memory deficit. Magnetic resonance imaging revealed multiple infra and supratentorial edematous and hemorrhagic lesions with restricted diffusion (Fig., Supplemental Digital Content 3, http://links.lww.com/INF/D234).

Extensive investigation failed to identify any infectious agent. PCR on multiple samples (blood, stools, respiratory secretions, cerebrospinal fluid [CSF], brain tissue) were negative for EBV, CMV, adenovirus, HHV6-8, enterovirus, HTLV, LCMV, HSV, VZV, parvovirus, JC and BK virus, arbovirus, bacterial 16s DNA, Rickettsia, Bartonella, Coxiella and Leptospira. Cultures from blood, stools, CSF, and brain biopsy were also negative. Examination of the $\mathrm{V} \beta \mathrm{T}$ cell repertoire in peripheral blood did not detect any clonal expansions (data not shown). A cerebral stereotactic biopsy revealed signs of vasculitis, with perivascular invasion by $\mathrm{CD}^{+} \mathrm{T}$ lymphocytes, without B-cells (Fig., Supplemental Digital Content 4, http://links.lww.com/INF/D235). Epstein-Barr encoding region staining for $\mathrm{EBV}$ in this biopsy was negative.

The patient received broad-spectrum antibiotics and acyclovir, as well as an aggressive treatment with intravenous immunoglobulin, high-dose steroids, rituximab and intrathecal methotrexate and dexamethasone. Nonetheless, his mental and motor condition worsened. One month after the first symptoms, he was unable to walk, talk or recognize his parents. He received cyclophosphamide and alemtuzumab but continued to deteriorate. Finally, the patient became comatose. There was a notion of neurologic irreversibility, so the patient did not receive HSCT. He ultimately died 4 months after the first neurologic symptoms.

\section{DISCUSSION}

We report an XLP1 patient who presented Burkitt lymphoma followed by CNS vasculitis at 14 years of age. His brother, who died of fulminant infectious mononucleosis at 2 years of age, likely carried the same mutation. Specific SH2D1A mutations generally present with a spectrum of manifestations without predictable genotype-phenotype correlations. ${ }^{5}$

Malignant lymphomas are a well known manifestation of XLP1, being the initial manifestation of the disease in $14 \%$ of the patients. ${ }^{1}$ Most of the cases arise in extranodal sites, with Burkitt lymphoma of the ileocecal region being the most common presentation. ${ }^{1,5,7}$ Remarkably, $20 \%$ of these patients are allegedly seronegative for $\mathrm{EBV}^{2} \mathrm{~A}$ lack of correlation between EBV infection and lymphoma can be explained by the incapacity of the SAP-deficient CD ${ }^{+}$cells to respond to B-cells. ${ }^{2}$ Recent studies have determined that $3 \%$ of male patients with B-cell non Hodgkin lymphoma harbour a $S H 2 D 1 A$ mutation. ${ }^{7}$

Central nervous system vasculitis is a very rare manifestation of XLP1. To our knowledge, there are only 8 previous patients with XLP1 manifesting central nervous system vasculitis (Table, Supplemental Digital Content 5, http://links.lww.com/INF/ D236) ${ }^{8-10}$ In the first descriptions, it was proposed that the vasculitis was a complication of EBV infection of the cerebral vessel walls (as a result of defective viral clearance) or by aberrant activation of cytotoxic $\mathrm{CD}^{+} \mathrm{T}$ cells by EBV-infected B cells. However, in subsequent reports, most of the cases had no evidence of ongoing EBV infection. The first 2 case reports to propose that CNS vasculitis could occur independent of active EBV infection did not perform PCR analysis of the CSF or brain tissue. In 2009, Talaat et $\mathrm{al}^{10}$ reported 2 patients with XLP1 that developed CNS vasculitis in the absence of ongoing EBV infection, substantiated by negative PCR in the affected tissues. We found EBV PCR repeatedly negative in the blood, CSF or brain tissue, as was Epstein-Barr encoding region in the brain tissue. Thus, our results substantiate the notion that severe manifestations of XLP1, including CNS vasculitis, may occur irrespective of EBV status.

Our report strengthens the idea that CNS vasculitis may occur irrespective of the EBV status of the recipient. The mechanisms that drive this fatal outcome in XLP1 patients remain obscure. Notably, reactivation-induced cell death is impaired in SAP-deficiency. ${ }^{2,5}$ Thus, we speculate that SAP-deficiency renders autoreactive $\mathrm{T}$ cells directed against vascular antigens resistant to apoptosis, potentially contributing to this rare clinical manifestation of XLP1.4,

Notably, our patient is the fourth (4/9) reported to develop CNS vasculitis after completing chemotherapy for Burkitt lymphoma. Opportunistic infections could be responsible for promoting aberrant immune responses, that is, CNS vasculitis. However, the fact that the abnormal cytotoxic responses in XLP1 are mostly related to antigens presented by $\mathrm{B}$ cells and that most pathogens are not restricted to these cells argues against this hypothesis. Moreover, we performed an extensive microbiologic investigation that was negative, which may rather suggest an aberrant response to endogenous ligands.

In summary, our report confirms that CNS vasculitis is a fatal manifestation of XLP1 that may occur in the absence of active EBV infection. Following diagnosis, HSCT should therefore be performed in a timely manner to avoid potentially fatal complications of the disease.

\section{REFERENCES}

1. Booth C, Gilmour KC, Veys P, et al. X-linked lymphoproliferative disease due to SAP/SH2D1A deficiency: a multicenter study on the manifestations, management and outcome of the disease. Blood. 2011;117:53-62.

2. Tangye SG. XLP: clinical features and molecular etiology due to mutations in SH2D1A encoding SAP. J Clin Immunol. 2014;34:772-779.

3. Cannons JL, Tangye SG, Schwartzberg PL. SLAM family receptors and SAP adaptors in immunity. Annu Rev Immunol. 2011;29:665-705. Available at http://www.annualreviews.org/doi/10.1146/annurev-immunol-030409-101302. Accessed December 17, 2017

4. Veillette A. Immune regulation by SLAM family receptors and SAP-related adaptors. Nat Rev Immunol. 2006;6:56-66

5. Filipovich AH, Zhang K, Snow AL, et al. X-linked lymphoproliferative syndromes: brothers or distant cousins? Blood. 2010;116:3398-3408.

6. Debeljak M, Podkrajsek KT, Aplenc R, et al. X-linked lymphoproliferative disease with a novel SH2D1A gene mutation. Pediatr Blood Cancer. 2008;50:187

7. Sandlund JT, Shurtleff SA, Onciu M, et al. Frequent mutations in SH2D1A (XLP) in males presenting with high-grade mature B-cell neoplasms. Pediatr Blood Cancer. 2013;60:E85-E87.

8. Verhelst H, Van Coster R, Bockaert N, et al. Limbic encephalitis as presentation of a SAP deficiency. Neurology. 2007;69:218-219.

9. Gray PE, O'Brien TA, Wagle M, et al. Cerebral vasculitis in X-linked lymphoproliferative disease cured by matched unrelated cord blood transplant J Clin Immunol. 2015;35:604-609.

10. Talaat KR, Rothman JA, Cohen JI, et al. Lymphocytic vasculitis involving the central nervous system occurs in patients with X-linked lymphoproliferative disease in the absence of Epstein-Barr virus infection. Pediatr Blood Cancer. 2009:53:1120-1123. 